Article

\title{
Long-Term Habitual Exercise and Combination of $\beta$-hydroxy- $\beta$-methylbutyrate Plus Black Ginger Maintain Muscle Health in SAMP8 Mice
}

\author{
Kai Aoki ${ }^{1 \dagger}$, Masaki Konno ${ }^{1 \dagger}$, Katsuyuki Tokinoya ${ }^{1}$, Katsunari Honda ${ }^{2}$, Takuya Abe ${ }^{3}$, Takeshi \\ Nagata $^{4}$, Masaaki Takehara ${ }^{5}$, Takehito Sugasawa ${ }^{6}$, Kazuhiro Takekoshi ${ }^{6}$, and Hajime Ohmori ${ }^{7 *}$ \\ 1 Graduate School of Comprehensive Human Sciences, University of Tsukuba, Tsukuba 1-1-1 Tennodai \\ 305-8577, Japan; s1930392@s.tsukuba.ac.jp (K.A.); konno.m.11017325@gmail.com (M.K.); ilbsk5@yahoo.co.jp \\ (K.T.) \\ 2 School of Physical Education, Health and Sport Sciences, University of Tsukuba, Tsukuba 305-8574, Japan; \\ khonda.iba1@gmail.com (K.H.) \\ 3 Zenyaku Hanbai Co., Ltd., Market and Product Development Department. 22-14, Higashiikebukuro \\ 3-Chome, Toshima-Ku, Tokyo 170-0013, Japan; Takuya_Abe@mail.zenyaku.co.jp (T.A.) \\ 4 Zenyaku Kogyo Co., Ltd, Healthcare Research Department of Drug Discovery, R\&D Center. 33-7, \\ Ohizumi-machi 2-Chome, Nerima-Ku, Tokyo 178-0062, Japan; Takeshi_Nagata@mail.zenyaku.co.jp (T.N.) \\ 5 Zenyaku Kogyo Co., Ltd, Consumer Product Planning Department. 6-15, Otsuka 5-Chome, Bunkyo-Ku, \\ Tokyo 112-8650, Japan; Masaaki_Takehara@mail.zenyaku.co.jp (M.T.) \\ 6 Faculty of Medicine, University of Tsukuba, 1-1-1 Tennodai, Ibaraki 305-8577, Japan; \\ take0716@krf.biglobe.ne.jp (T.S.); k-takemd@md.tsukuba.ac.jp (K.T.) \\ 7 Faculty of Health and Sport Sciences, University of Tsukuba, Tsukuba 305-8574, Japan \\ *Correspondence: omori.hajime.gb@u.tsukuba.ac.jp; Tel.: +81-02-9853-2676 \\ † These authors contributed equally to this work.
}

Received: date; Accepted: date; Published: date

\begin{abstract}
Muscle mass and strength decrease with aging, but habitual exercise can maintain muscle health. $\beta$-Hydroxy- $\beta$-methyl butyrate calcium (HMB) and black ginger (BG) are anti-oxidants that have been reported to improve muscle protein metabolism and energy production; these molecules may have synergistic effects. The senescence-accelerated mouse-prone 8 (SAMP8) model is a useful model of muscle aging. Therefore, in this study, we explored how the combination of habitual exercise, $\mathrm{HMB}$, and BG affected muscle aging. We used 28-week-old SAMP8 mice divided into five groups: control, exercise (Ex), Ex+BG, Ex+HMB, and $\mathrm{Ex}+\mathrm{BG}+\mathrm{HMB}(\mathrm{Ex}+\mathrm{Comb})$. Mice were required to run on a treadmill for 16 weeks at 5 days per week. In 44-week-old mice, grip strength tests and dissection were conducted. Muscle weight was measured, and the gastrocnemius muscle was subjected to quantitative polymerase chain reaction and immunoblotting. Muscle mass and strength were preserved in the Ex+Comb group, and mitochondrial function was preserved through suppressing oxidative stress. Muscle protein synthesis signaling was improved in the Ex+Comb group. Autophagy and the ubiquitin system were normalized by Ex+Comb treatment. Overall, habitual exercise and HMB plus BG treatment maintained muscle health by suppressing oxidative stress, preserving mitochondrial function, and maintaining muscle protein metabolism in SAMP8 mice.
\end{abstract}

Keywords: Sarcopenia; $\beta$-hydroxy- $\beta$-methyl butyrate calcium; black ginger

\section{Introduction}

Aging affects various organs, including the brain, blood vessels, bone, digestive tract, and skeletal muscle. Skeletal muscle aging is called sarcopenia; this process is characterized by loss of muscle mass and strength. Sarcopenia was initially described by Rosenberg [1]. The European Working Group on Sarcopenia in Older People recommend using both the loss of skeletal muscle mass and the loss of muscle function for diagnosis of sarcopenia. This definition is complex because 
muscle strength is dependent not only on muscle mass but also on function. The etiology of sarcopenia is multifaceted. For example, reactive oxygen species (ROS) have been shown to be increased in elderly people with sarcopenia [2]. Moreover, excessive ROS harms the mitochondria and causes mitochondrial dysfunction [3]. Importantly, mitochondrial functions, such as ATP synthesis, are decreased with age-induced increases in ROS, resulting in muscle weakness [4]. In addition, mitochondria and skeletal muscle cells are closely linked and together regulate muscle mass. Namely, maintaining mitochondrial function is important for maintaining muscle mass [5]. Similarly, the balance between protein synthesis and protein degradation is important for maintaining muscle mass. Protein synthesis decreases with aging, whereas protein degradation increases with aging. However, recent studies have suggested that changes in the activity of protein degradation systems, such as the ubiquitin proteasome system (UPS) and autophagy, are not beneficial for muscle health [6-8]. Furthermore, excessive decreases in protein degradation functions induce accumulation of unnecessary protein, resulting in decreased muscle strength [9]. Therefore, to maintain muscle mass, muscle degradation systems must be normalized while maintaining muscle synthesis signals.

To avoid the muscle weakness related to muscle mass loss and muscle strength decline, various approaches have been attempted. Habitual exercise has positive effects on maintaining muscle strength [10-15]. Although aging affects ROS production and mitochondria function, habitual exercise can protect against these changes to maintain mitochondrial function and regulate mitochondrial quality or content $[13,14]$. Moreover, Fujita reported that exercise suppresses decreases in Akt phosphorylation and enhances muscle protein synthesis signals [15]. Additionally, aging can cause dysfunction of autophagy, whereas exercise can attenuate this process [12].

In addition to exercise, ingestion of functional components can also be used to block muscle weakness. $\beta$-Hydroxy- $\beta$-methyl butyrate calcium (HMB) is a metabolite of leucine and has been reported to have strong muscle synthesis effects, thereby inhibiting muscle protein degradation. Kimura reported that HMB stimulates mammalian target of rapamycin (mTOR) via the phosphatidylinositol 3-kinase/Akt signaling pathway and suppress the muscle-specific ubiquitin E3 ligase MuRF1 [8]. Furthermore, HMB suppresses excessive autophagy [16]. HMB has also been reported to be involved in mitochondrial biogenesis, muscle protein synthesis, and muscle protein degradation. Stancliffe reported that HMB stimulates mitochondrial biogenesis regulators, such as peroxisome proliferator-activated receptor gamma coactivator 1-alpha (PGC-1 $\alpha$ ), consequently increasing mitochondria biogenesis in $\mathrm{C} 2 \mathrm{C} 12$ myotubes [17]. Kaempferia parviflora (black ginger [BG]) has multiple effects, including antiglycation, anti-inflammatory, anti-oxidant, and energy production-enhancing effects via increasing mitochondrial activity [18-21]. In particular, Wattanathorn reported that administration of BG for 8 weeks enhances physical performance by decreasing oxidative stress in healthy elderly individuals [22]. Therefore, these supplements may be used to maintain muscle health via various molecular mechanisms.

Some animal models have been developed to study aging because animal models of natural aging require a lot of time for evaluations. Senescence-accelerated mice (SAMs) are a commonly used animal model of aging established by Takeda [23]. There are eight strains of SAMs. In particular, SAMP8 mice have been used to study muscle aging [24, 25]. In our previous studies, we demonstrated that muscle mass and strength were decreased from 28 to 44 weeks of age (data not shown).

Accordingly, we hypothesized that long-term habitual exercise and a combination of HMB plus BG may improve muscle health in SAMP8 mice by enhancing mitochondrial function, increasing muscle protein synthesis, and normalizing muscle protein degradation. 


\section{Materials and Methods}

\subsection{Animal treatment and grouping}

Animal experiments in this study were approved by the Animal Care Committee, University of Tsukuba (approval number: 17-414). An overview of the experiments is shown in Figure. 1. Male SAMP8 mice aged 28 weeks old (Japan SLC, Hamamatsu, Japan) were kept at 20-26 ${ }^{\circ} \mathrm{C}$ with a humidity of 40-60\% under a 12-h light/dark cycle under conventional conditions. Mice were freely fed a standard diet (Oriental Yeast, Tokyo, Japan) containing appropriate supplements. Mice were then divided into five groups: sedentary control group (Con), exercise for 16 weeks (Ex), Ex and HMB intake (Ex+HMB), Ex and BG $(E x+B G)$, and Ex and HMB plus BG (Ex+Comb), with eight mice in each group.

28w-old

44w-old

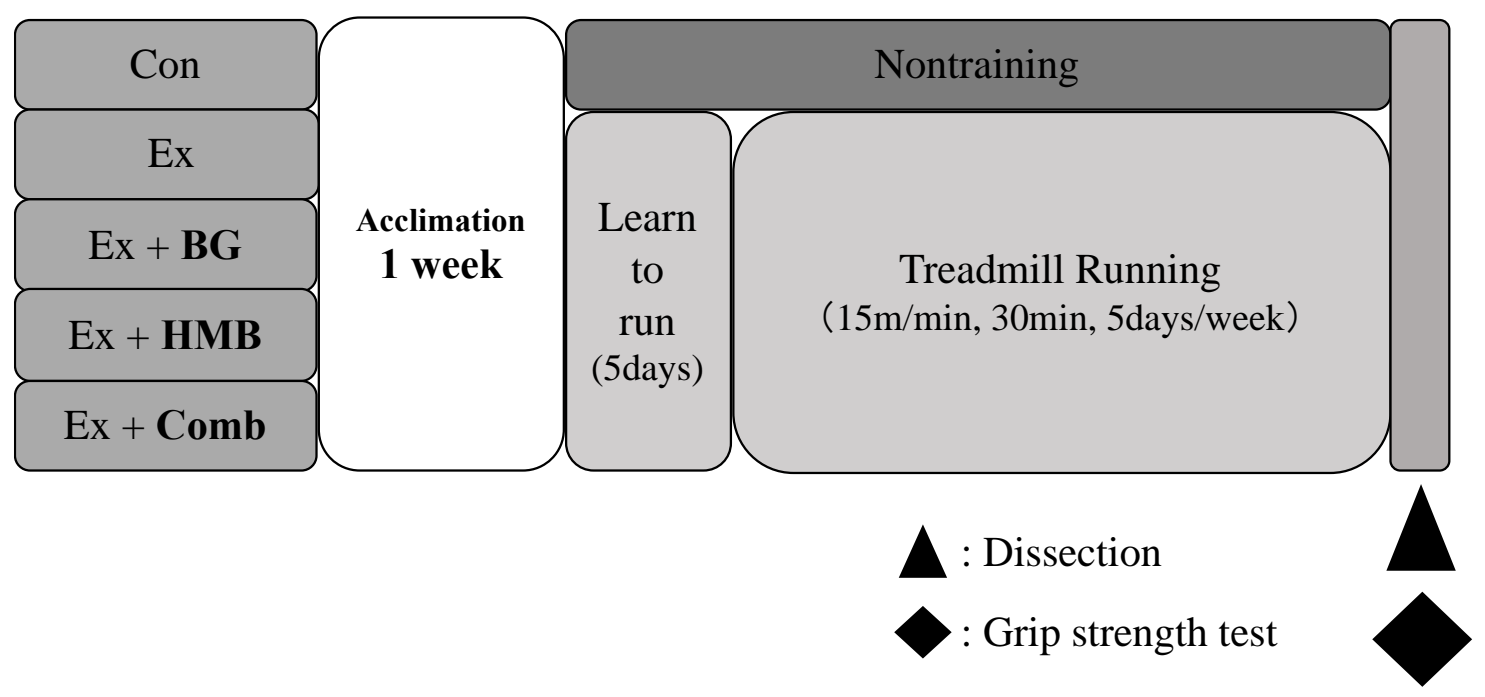

Figure 1. Overview of the study design

\subsection{Supplement preparation}

Recommended intakes of HMB (Kobayashi Perfumery Co., Ltd., Tokyo, Japan) and BG (Maruzen Pharmaceuticals Co., Ltd., Hiroshima., Japan) in humans are 1.5 g/day and 90 mg/day, respectively $[22,26]$. We converted the recommended amounts in humans to animals for BG; however, because the taste of HMB is bitter, one-third of the amount (500 mg) was converted for continuous intake. HMB was mixed with powdered feed at $83.3 \mathrm{mg} / \mathrm{kg} / \mathrm{day}$; BG was mixed with powdered feed at $15 \mathrm{mg} / \mathrm{kg} /$ day; and the combined treatment $(\mathrm{HMB}+\mathrm{BG})$ was mixed with powdered feed to combined the concentrations of both components.

\subsection{Treadmill running training protocol}

Animals in exercise groups were made to run on a treadmill made for small animals (FVRO, 4E9S-6; Fuji Medical Science, Chiba, Japan). Animals were allowed to learn how to run for 5 days. Subsequently, training was performed at $15 \mathrm{~m} / \mathrm{min}$ for 5 days a week at $30 \mathrm{~min} /$ day from 28 weeks of age to 44 weeks of age. Training was conducted from 08:00 to 13:00.

\subsection{Four-limb grip strength test}

Grip strength tests were performed for all four limbs using a small animal grip measurement device (GMP-100B; MELQUEST, Toyama, Japan) as a muscle strength index at 44 weeks of age. Mice were held such that their limbs were gripping the device, and the tail was pulled backward. The 
maximum value of the force applied to release the grip was used as the measurement value. The measurement was performed five times, and the average value, excluding maximum and minimum values, was taken as the value for each individual. In addition, the same examiner made the measurement every time. Measurements were made such that the examiner would not know the measured value or which individual was being tested in order to avoid bias.

\subsection{Dissection and sampling}

At the time of investigation (44 weeks old; Figure 1), mice were anaesthetized by an intraperitoneal injection of pentobarbitone sodium (Kyoritsu Seiyaku Corporation, Tokyo, Japan). Soleus muscles, plantaris muscles, and gastrocnemius muscles were harvested, and mice were killed by cervical dislocation. The muscle wet weights were measured at the same time. Muscle samples were quickly frozen in liquid nitrogen and stored at $-80^{\circ} \mathrm{C}$ for subsequent biochemical assays.

\subsection{Reverse transcription quantitative polymerase chain reaction (RT-qPCR)}

Total RNA was extracted from the gastrocnemius muscle using Sepasol-RNA I Super G (Nacalai Tesque, Kyoto, Japan). The total RNA concentration was measured with a spectrophotometer (NanoDrop 2000; Thermo Fisher Scientific, MA, USA). Based on the values obtained, the samples were adjusted to $400 \mathrm{ng} / \mu \mathrm{L}$ with sterile water. After adding $5 \times$ PrimeScript RT Master Mix (RR 036 A; Takara Bio, Shiga, Japan) and RNase-free water to the diluted RNA, reverse transcription was carried out in a thermal cycler (TP 350; Takara Bio; $\left(37^{\circ} \mathrm{C}, 15 \mathrm{~min} ; 85^{\circ} \mathrm{C}\right.$, $\left.5 \mathrm{~s} ; 4^{\circ} \mathrm{C}, \infty\right)$. After reverse transcription, a KAPA SYBR FAST qPCR kit (Kapa Biosystems, Wilmington, MA, USA) was used for qPCR. RT-qPCR was performed using an ABI 7500 Fast system (Applied Biosystems, Foster City, CA, USA). The cycle threshold $(\mathrm{Ct})$ values of the target genes were standardized according to the $\mathrm{Ct}$ value of the housekeeping gene $(\Delta \Delta \mathrm{Ct}$ method). The relative expression levels of the target genes were calculated as the relative value compared with that of the control group. The sequences of primers used in this study are shown in Table 1.

Table 1. List of primers used in this study.

\begin{tabular}{|c|c|c|}
\hline Gene name & Primer sequence & bp \\
\hline \multirow{12}{*}{$\begin{array}{c}\text { Citrate synthesis } \\
(C S) \\
\text { Cytochrome } \mathrm{c} \text { oxidase subunit } 4 \\
(C O X \mathrm{IV})\end{array}$} & 5'-TGCTCTAATCAGGACCATTG-3' & \multirow[t]{2}{*}{81} \\
\hline & 5'-GTAGTAAGCGTGCTCCCACAC-3' & \\
\hline & 5'-CTCTTGGGAGAGCCTGACA-3' & \multirow{2}{*}{73} \\
\hline & 5'-GCCAGTAGCAAGCCGTAGAA-3' & \\
\hline & 5'-CCTTCAAGTTGGTTTAATGCAGA-3' & \multirow{2}{*}{80} \\
\hline & 5'-CAAGTTTTTGATGCCCTGGT-3' & \\
\hline & 5'-CTGCACCCTATGTCTCAGCTC-3' & \multirow{2}{*}{132} \\
\hline & 5'-GAGGTCCATGCAGTCCTCA-3' & \\
\hline & 5'-TGATGTGGCCCATGTCAC-3' & \multirow{2}{*}{121} \\
\hline & 5'-GTTAAACTGGATGCGGTACAACT-3' & \\
\hline & 5'-TCCATGCCTCTAACACTCGAC-3' & \multirow{2}{*}{122} \\
\hline & 5'-GCTTAACACACGCCCAGTCT-3' & \\
\hline PPAR gamma coactivator 1 -alpha & 5'-GCGAACCTTAAGTGTGGAACTC-3' & \multirow{2}{*}{93} \\
\hline$(P G C-1 \alpha)$ & 5'-GCCTTGAAAGGGTTATCTTGG-3' & \\
\hline Transcription factor A, mitochondrial & 5'-AGGGAGCTACCAGAAGCAGA-3' & \multirow{2}{*}{119} \\
\hline$(T F A M)$ & 5'-CCCATCAGCTGACTTGGAGT-3' & \\
\hline Tripartite motif-containing 63 & 5'-ACTGCATCTCCATGCTGGT-3' & \multirow{2}{*}{106} \\
\hline$(M u R F 1)$ & 5'-TCCTTCACCTGGTGGCTATT-3' & \\
\hline F-box protein 32 & 5'-CAGCAGCAGCTGAATAGCAT-3' & \multirow{2}{*}{80} \\
\hline$(M A F b x)$ & 5'-TGTAAGCACACAGGCAGGTC-3' & \\
\hline TATA box binding protein & 5'-CCAATGACTCCTATGACCCCTA-3' & \multirow{2}{*}{104} \\
\hline$(T b p)$ & 5'-CAGCCAAGATTCACGGTAGAT-3' & \\
\hline
\end{tabular}

\subsection{Immunoblotting analysis}

Total proteins were extracted from gastrocnemius muscles with RIPA buffer (1\% NP-40, 0.1\% sodium dodecyl sulfate [SDS], $20 \mathrm{mM}$ Tris- $\mathrm{HCl}$ [pH 8.0], $5 \mathrm{mM}$ ethylenediaminetetraacetic acid, 150 $\mathrm{mM} \mathrm{NaCl}$ plus proteinase inhibitor tablets [cOmplete mini; Roche, Basel, Switzerland] and 
phosphatase inhibitor [PhosSTOP; Roche]). The lysate were centrifuged at 12,000 $\times g$ for $15 \mathrm{~min}$ at $4^{\circ} \mathrm{C}$, and the supernatant was used for subsequent analyses. The total protein concentration of each sample was measured using a BCA protein assay kit (Takara Bio). Next, $20 \mu \mathrm{g} / \mathrm{lane}$ total protein was used for SDS-polyacrylamide gel electrophoresis. After electrophoresis, gels were transferred to polyvinylidene difluoride membranes (WSE-4053; ATTO, Tokyo, Japan), and specific antibodies were used for detection of target proteins. The antibodies used in this study are shown in Table 2. Signals were detected using chemiluminescence reagent (SignalFireTM Elite ECL Reagent; Cell Signaling Technology, Danvers, MA, USA). Detected images were scanned using a Light-Capture Cooled CCD Camera System (Image Quant LAS-4000; GE Healthcare, UK).

Table 2. List of antibodies used in this study.

\begin{tabular}{cccc}
\hline Product code & Protein name & Species & Dilution \\
\hline CST \#4691T & Akt & Rabbit & $1: 2000$ \\
CST \#4060T & Phospho-Akt (Ser473) & Rabbit & $1: 2000$ \\
CST \#2708T & p70S6K & Rabbit & $1: 2000$ \\
CST \#9205S & Phospho-p70S6K(Thr389) & Rabbit & $1: 2000$ \\
CST \#2217 & Ribosomal protein S6 & Rabbit & $1: 2000$ \\
CST \#2211 & Phospho-ribosomal protein S6 (Ser235/236) & Rabbit & $1: 2000$ \\
CST \#9452 & 4E-BP1 & Rabbit & $1: 2000$ \\
CST \#9455 & Phospho-4E-BP1 (Thr70) & Rabbit & $1: 2000$ \\
Abcam ab168372 & F-bx32 (MAFbx) & Rabbit & $1: 2000$ \\
Abcam ab172470 & MuRF1 & Rabbit & $1: 2000$ \\
CST \#3415T & Atg3 & Rabbit & $1: 2000$ \\
CST \#8089T & Atg16L1 & Rabbit & $1: 2000$ \\
CST \#3495T & Beclin-1 & Rabbit & $1: 2000$ \\
CST \#8558T & Atg7 & Rabbit & $1: 2000$ \\
CST \#12741 & LC3A/B & Rabbit & $1: 2000$ \\
CST \#7074 & Anti-rabbit IgG, HRP-linked antibody & Rabbit & $1: 2000$ \\
\hline
\end{tabular}

\subsection{Statistical analysis}

Data are shown as means \pm standard errors of the means (SEMs). Statistical analyses were performed using SPSS statistics ver. 26 for Mac (SPSS Japan, Tokyo, Japan).

\section{Results}

3.1 Habitual exercise and combination of BG plus HMB preserved muscle mass and strength and decreased white adipose tissue (WAT) weight

Muscle mass and strength decline beginning at 28 weeks of age in SAMP8 mice [25]. Additionally, we confirmed that muscle mass and strength were decreased with aging in SAMP8 mice (unpublished data). Therefore, to evaluate the effects of habitual exercise and a combination of BG plus HMB on muscles, we measured skeletal muscle mass and muscle strength. In the Ex+HMB group, plantaris muscle mass was higher than that in the Con group. Moreover, in the Ex+Comb group, all muscle masses and grip strength were significantly higher than those in the Con group. In WAT was significantly lower in the Ex+Comb group than in the Con group. 
A)
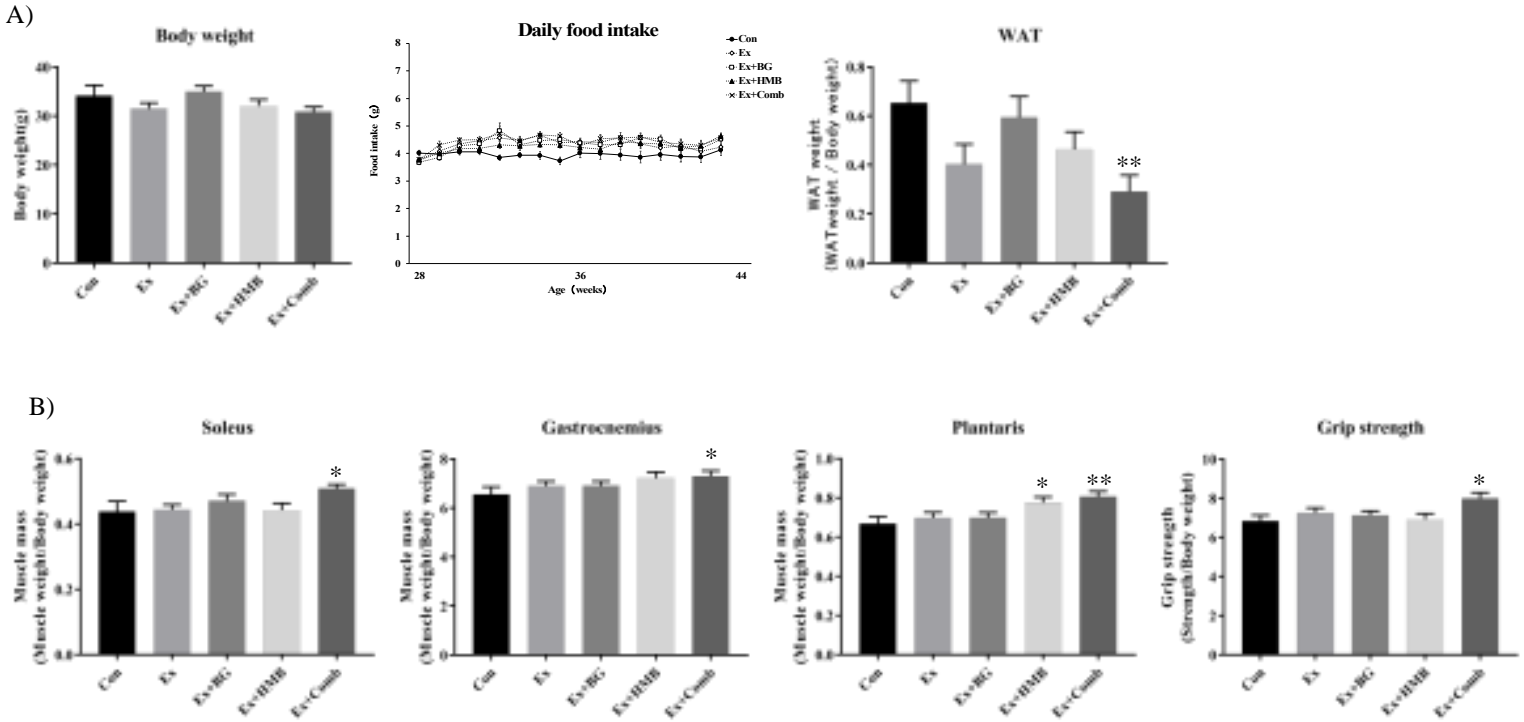

Figure 2. Effects of habitual exercise and combination of BG plus HMB on basic health data in 44-week-old mice. A) Body weight, daily food intake, and white adipose tissue weight at 44 weeks old in SAMP8 mice. Body weight was measured before dissection. White adipose tissue was harvested from each mouse by dissection, and wet weight was measured. Daily food intake was measured once a week. B) Muscle wet weight for each type of muscle and grip strength at 44 weeks of age in SAMP8 mice. Soleus, gastrocnemius, and plantaris muscles were harvested from mice, and wet weight was measured. Grip strength was measured before dissection. The measurement was performed five times, and the average value, excluding the maximum and minimum values, was used for each individuals. Values indicate means \pm SEMs $(n=8)$. Asterisks indicate significant differences compared with the control group (Con; ${ }^{*} p<0.05$, ${ }^{* *} p<0.01$ ).

\subsection{Oxidative stress was suppressed by a combination of habitual exercise and BG plus HMB supplementation}

Oxidative stress increases with aging, and the levels of antioxidant enzymes increase in order to suppress oxidative stress. Consequently, we evaluated the expression levels of antioxidant stress enzyme genes in gastrocnemius muscles. For superoxide dismutase 2 (SOD2), there were no changes in all groups. However, for SOD3, expression tended to be lower in the Ex+BG $(p=0.09)$ and Ex + Comb groups $(p=0.08)$. Catalase expression also tended to be lower in the Ex+Comb group $(p=$ $0.1)$.
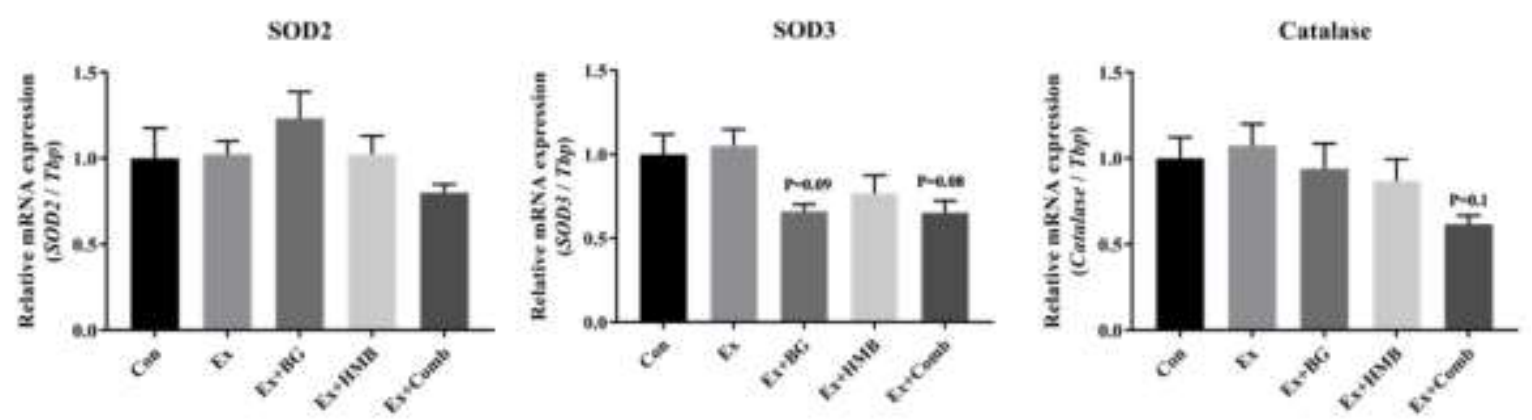

Figure 3. Effects of habitual exercise and combination of BG plus HMB on the expression levels of antioxidant enzyme genes. RNA was extracted from gastrocnemius muscles, converted to cDNA, and used as a template for RT-qPCR. SOD2: superoxide dismutase 2, SOD3: superoxide dismutase 3. Relative mRNA expression levels for each gene were normalized to the mRNA expression of TATA box binding protein $(T b p)$. Values indicate means \pm SEMs $(\mathrm{n}=7-8) . P$ values in the graph indicate significant trends compared with the control group (Con). 
3.3 The expression levels of mitochondrial function- and biogenesis-related genes and mitochondrial oxidative phosphorylation (OXPHOS) complex were increased by a combination of habitual exercise and BG plus HMB supplementation

Mitochondrial function and biogenesis decrease with aging and are related to sarcopenia. Therefore, we next examined the effects of habitual exercise and BG plus HMB supplementation on mitochondrial function- and biogenesis-related genes. Citrate synthase expressed tended to be higher in the Ex+Comb group $(p=0.068)$, whereas cytochrome c oxidase subunit IV showed significant higher in the Ex+HMB $(p<0.05)$ and $\mathrm{Ex}+\mathrm{Comb}$ groups $(p<0.01)$. ATP synthase expression significant higher in the Ex+Comb group $(p<0.01)$. Moreover, PGC-1 $\alpha$ expression showed significant higher in the Ex+HMB $(p<0.05)$ and Ex+Comb groups $(p<0.01)$. Mitofusion $(M f n) 1$, $M f n 2$, and optic atrophy 1 (Opa1), which are mitochondrial fusion markers, showed significant higher in the Ex+Comb group $(p<0.01)$.

Mitochondria OXPHOS is an index for mitochondrial function. Therefore, we next measured protein expression levels of components of the OXPHOS complex. There were significant higher in the expression levels of complex I and II in the Ex+Comb group $(p<0.05)$. An higher trend was also observed for complex $\mathrm{V}$ in the Ex+Comb group ( $p=0.057)$.

A)

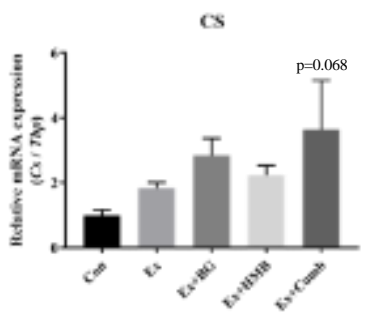

PGC-1a
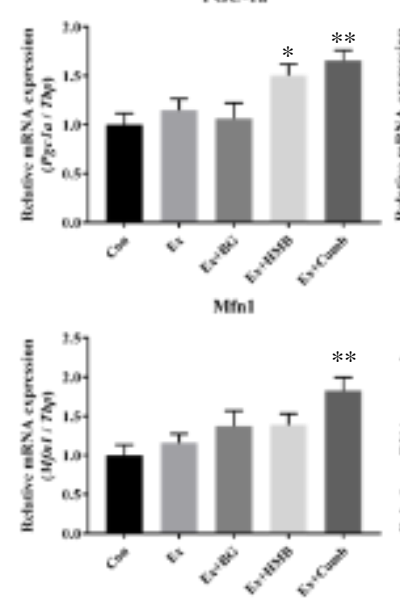

B)
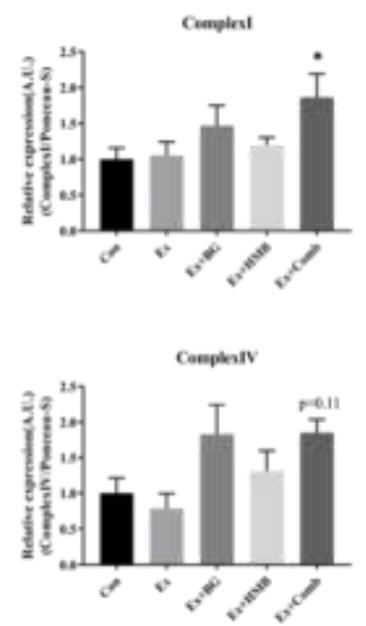

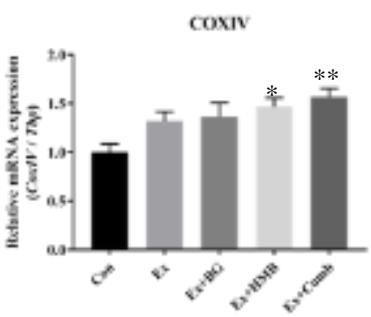

TFAM

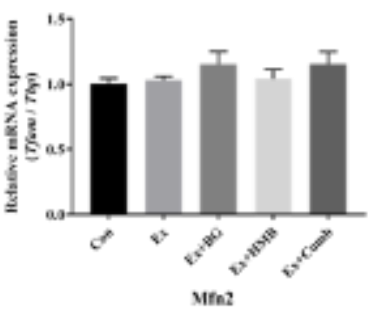

Mte?
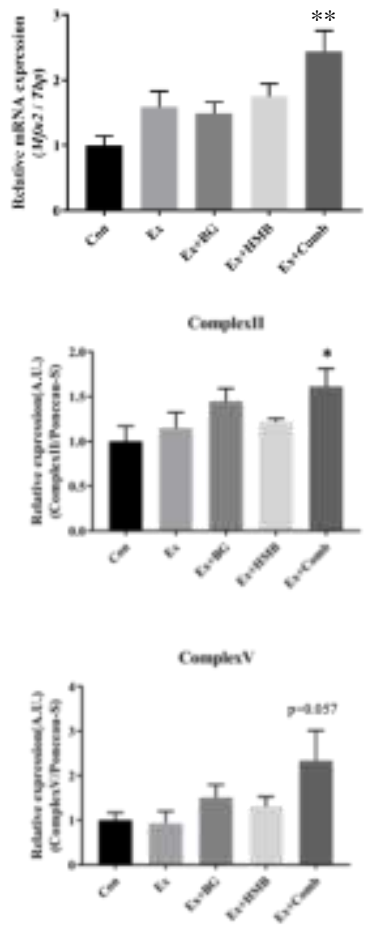

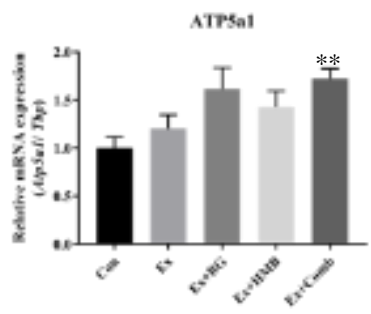

Mir
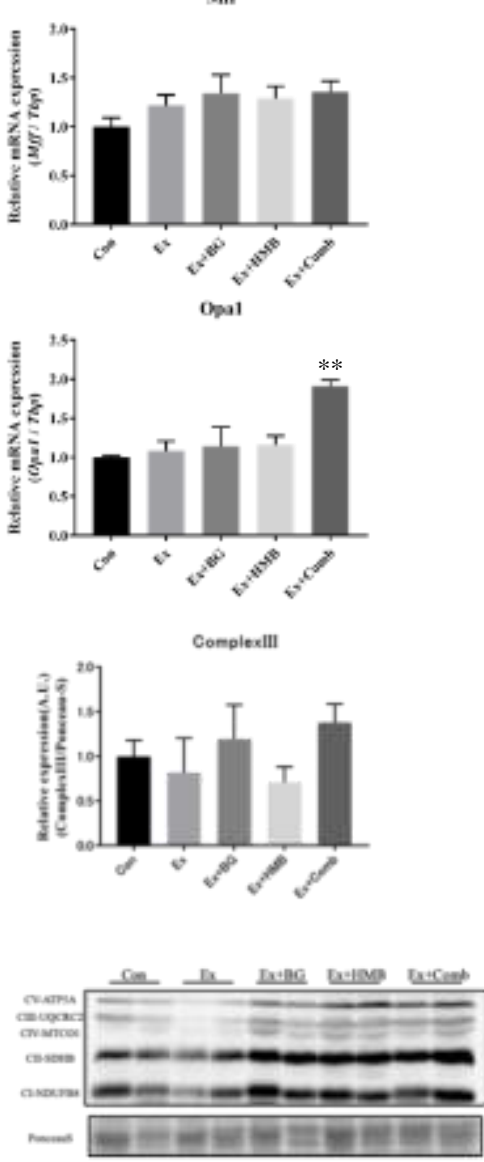
Figure 4. Effects of habitual exercise and combination of BG plus HMB on mitochondrial biogenesis and function. A) Mitochondrial function- and biogenesis-related genes. RNA was extracted from gastrocnemius muscles, converted to cDNA, and used as template for RT-qPCR. Values indicate means \pm SEMs $(n=8)$. Asterisks indicate significant differences compared with the control group (Con; $\left.{ }^{*} p<0.05,{ }^{* *} p<0.01\right)$. CS: citrate synthesis; COXIV: cytochrome c oxidase subunit IV; ATP5a1: ATP synthase, $\mathrm{H}+$ transporting, mitochondrial $\mathrm{F} 1$ complex, alpha subunit $1 ;$ PGC-1 $\alpha$ : PPAR gamma coactivator 1-alpha; TFAM: transcription factor A, mitochondrial; Mff: mitochondrial fission factor; Mfn1: mitofusion1; Mfn2: mitofusion2; Opa1: optic atrophy 1. The relative mRNA expression level of each gene was normalized to the expression of TATA box binding protein (Tbp) mRNA. B) Mitochondria OXPHOS complex. Total protein was extracted from gastrocnemius muscles, and all proteins were analyzed by immunoblotting analysis. CI: Complex I-ATP5A, CII: Complex II-UQCRC2, CIII: Complex III-UQCRC2, CIV: Complex IV-MTCO1, CV: Complex V-ATP5A. Values indicate means \pm SEMs $(n=6)$. Asterisks indicate significant differences compared with the control group (Con; $\left.{ }^{*} p<0.05,{ }^{* *} p<0.01\right)$.

\subsection{Habitual exercise and HMB plus BG supplementation upregulated muscle protein synthesis signaling}

Muscle protein synthesis signaling decreases with aging and causes muscle protein metabolism to be unbalanced. We confirmed the effects of habitual exercise and BG plus HMB supplementation on protein phosphorylation levels of protein synthesis signaling-related proteins. The levels of Akt were significant higher in the Ex+Comb group $(p<0.05)$. Additionally, RpS6 expression tended to be higher in the Ex+Comb group $(p=0.107)$.

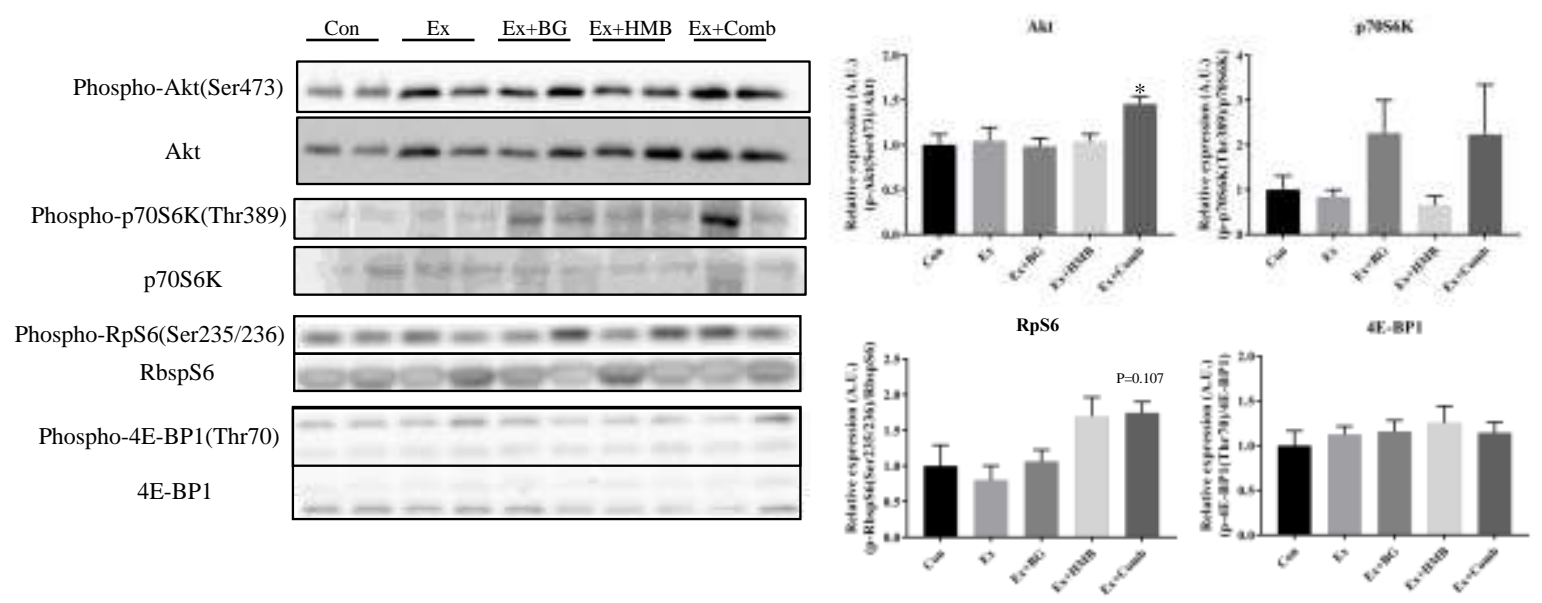

Figure 5. Effects of habitual exercise and combination of BG plus HMB on the expression levels of muscle protein synthesis-related proteins. Total protein was extracted from gastrocnemius muscles, and all proteins were analyzed by immunoblotting analysis. Akt: protein kinase B, p70S6K: 70-kDa ribosomal protein S6 kinase, RpS6: ribosomal protein S6, 4E-BP1: eukaryotic translation initiation factor 4E-binding protein 1. Relative levels of phosphorylated proteins were normalized to the expression levels of total proteins. Values indicate means \pm SEMs $(n=6-8)$. Asterisks indicate significant differences compared with the control group (Con; $\left.{ }^{*} p<0.05\right)$, and $P$ values in the graphs indicate significant changes compared with the control group (Con).

3.5 Muscle-specific E3 ubiquitin ligase was downregulated by a combination of habitual exercise and HMB plus BG supplementation

The effects of aging on muscle-specific E3 ubiquitin ligase are inconsistent. Therefore, we examined the expression levels of proteins and genes. For MuRF1, a significant lower trend was observed in the Ex+Comb group $(p=0.081)$. There were no changes in mRNA expression levels of both genes. 
A)
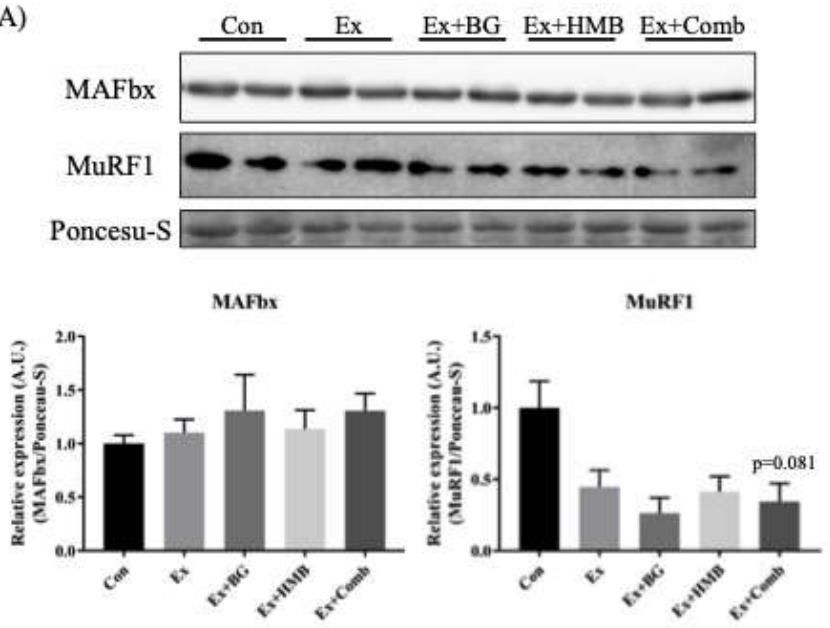

B)
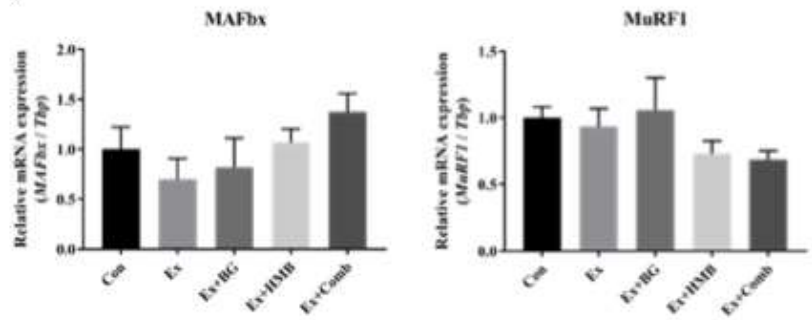

Figure 6. Effects of habitual exercise and combination of BG plus HMB on the expression levels of muscle-specific E3 ubiquitin ligase. A) Expression levels of muscle-specific E3 ubiquitin ligase proteins. B) Expression levels of muscle-specific E3 ubiquitin ligase genes. MAFbx: muscle atrophy F-box protein, MuRF1: muscle RING finger protein 1. Total protein was extracted from gastrocnemius muscles, and all proteins were analyzed by immunoblotting. Values indicate means \pm SEMs $(n=6)$. RNA was extracted from gastrocnemius muscles, converted to cDNA, and evaluated by RT-qPCR. The relative mRNA expression level of each gene was normalized to the mRNA expression of TATA box binding protein $(T b p)$. Values indicate means \pm SEMs $(\mathrm{n}=7-8)$.

3.6 Autophagy functions were stabilized by a combination of habitual exercise and HMB plus BG supplementation

Autophagy functions are affected by aging; however, the expression levels of autophagy-related proteins are inconsistent. Hence, we examined the effects of habitual exercise and BG plus HMB supplementation on autophagy function-related proteins. Atg3 and Atg7 expression levels were significant lower in the Ex+Comb group (Atg3: $p=0.05$, Atg7: $p=0.01$ ). For Atg16L1 and Beclin1, there were significant higher in the Ex+HMB group $(p<0.05)$ and Ex+Comb group $(p<0.01)$. 


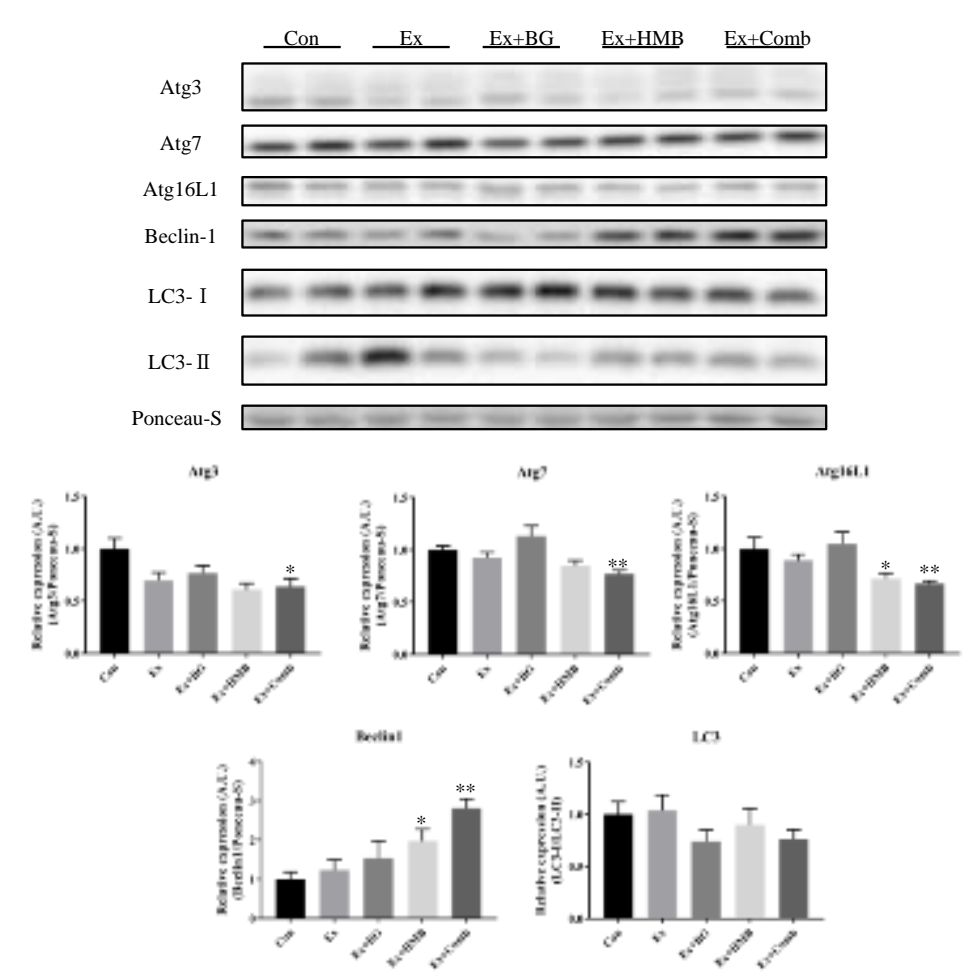

Figure 7. Effects of habitual exercise and combination of BG plus HMB on the expression levels of autophagy-related proteins. Total protein was extracted from gastrocnemius muscles, and all proteins were analyzed by immunoblotting. Atg3: autophagy-related protein 3, Atg7: autophagy-related protein 7, Atg16L1: autophagy-related protein 16L1, Beclin1: coiled-coil myosin-like BCL2-interacting protein, LC3-I: light chain 3-I, LC3-II: light chain 3-II. Values indicate means \pm SEMs $(n=7-8)$. Asterisks indicate significant differences versus the control group (Con; ${ }^{*} p<$ $\left.0.05,{ }^{* *} p<0.01\right)$.

\section{Discussion}

In the current study, we investigated the effects of long-term habitual exercise and combination of HMB plus BG supplementation on muscle health using SAMP8 mice. Our data showed that the combination of habitual exercise and supplementation maintained muscle health through reduction of oxidative stress, improvement of mitochondrial function, and modulation of muscle protein synthesis and degradation functions.

Some studies have reported SAMP8 mice are a useful model for muscle aging, including sarcopenia. In particular, Guo et al. reported that muscle mass in SAMP8 mice peaked at 7 months of age and that muscle strength decline began at 8 months of age [25]. In addition, we confirmed that muscle mass and muscle strength decreased from 28 to 44 weeks of age in SAMP8 mice (data not shown); therefore, we used SAMP8 mice at 28 weeks of age.

In this study, WAT weights were lowest in the Ex+Comb group; however, there were no differences in body weight within all groups. Thus, the combined effects of exercise and both supplements maintained muscle weight and reduced excess fat. In a previous study, HMB and BG were shown to reduce body fat mass $[27,28]$. Compared with these previous results, intake of supplements in this study was not effective alone owing to the lower volume; however, the combination increased the fat-reducing effect and caused fat loss. Fat accumulation is associated with excessive production of inflammatory cytokines, which can be a factor in sarcopenia. Additionally, the production of inflammatory cytokines may have been suppressed because fat was reduced by the combined use of exercise and supplements in this study. However, we did not measure the levels of blood inflammatory cytokines; thus, further studies are needed to investigate these factors. 
In this study, the Ex+Comb exhibited the highest was muscle mass and grip strength; thus, mitochondrial function and muscle protein synthesis/degradation signals were examined. Aging can cause high of oxidative stress [2]. In this study, we showed that SOD3 and catalase mRNA levels in tended to be lower in the Ex+BG and Ex+Comb groups. The expression of anti-oxidant enzymes has been shown to decrease when oxidative stress is low or when antioxidants are ingested. BG has antioxidant ability; accordingly, this decline in SOD3 and catalase levels suggested that oxidative stress levels may be decreased by BG. Excessive oxidative stress damages mitochondria and impairs mitochondrial function. Indeed, we observed increases in mitochondrial function- and biogenesis-related genes and mitochondrial OXPHOS complex expression in the $\mathrm{Ex}+\mathrm{Comb}$ group compared with that in the Con group. These results were consistent with the results of antioxidant enzyme gene expression. In contrast, BG and HMB have been reported to enhance mitochondrial biosynthesis $[17,21]$. In particular, BG and HMB upregulate the expression of PGC-1 $\alpha$, a master regulator of mitochondrial biosynthesis. In addition, our findings indicated that Mfn1, Mfn2, and Opa1, mitochondria fusion factors, were upregulated in the Ex+Comb group compared with that in the Con group. Mitochondrial fusion is decreased during aging and can cause mitochondria dysfunction. Therefore, combination of BG and HMB plus habitual exercise could maintain mitochondrial fusion during aging. Consistent with these results, the expression levels of OXPHOS complexes, an indicator of mitochondrial function, were highest in the Ex+Comb group. Some studies of BG and HMB have focused on mitochondrial biogenesis factors; however, few studies have evaluated mitochondrial dynamics. A recent study suggested that normal mitochondria dynamics contributed the muscle remodeling [5]. Therefore, the results of this study provide important insights into this process.

During muscle protein synthesis/degradation signaling, phosphorylation of Akt, which is upstream of the protein synthesis signal, was higher in the Ex+Comb group than in the Con group. In addition, the phosphorylation of RpS6, which acts downstream of the protein synthesis signal, tended to be higher in the Ex+Comb group. Because the exercise protocol conducted in this study could be performed in humans, even at an old age, the exercise protocol was established at an exercise intensity of moderate or low. Habitual exercise is known to enhance muscle synthesis by improving insulin sensitivity; however, we did not observe such changes induced by exercise intensity in this study. Notably, even with this exercise intensity, it was possible to enhance the muscle synthesis signal by administration of BG and HMB together. Similarly, the protein expression levels of MuRF1, a muscle-specific ubiquitin ligase that also acts on protein degradation, tended to decrease, even with exercise alone, and further decreases were observed with supplementation. Transcription of MuRF1 and MAFbx is regulated by the transcription factor Forkhead box O1 (Foxo1) [29]. Foxo1 translocates into the nucleus and regulates the transcription of both proteins, and phosphorylation of Akt stimulates the phosphorylation of Foxo1, resulting in inhibition of nuclear translocation and suppression of muscle protein degradation. In the current study, exercise and supplementation increased Akt phosphorylation and inhibited Foxo1 translocation into the nucleus, thereby suppressing MuRF1 expression and predominantly promoting muscle synthesis signals. MAFbx may be differentially regulated at the transcriptional level compared with MuRF1, consistent with our findings of the effects of exercise and supplements in this study. Overall, the muscle synthesis system was superior to the muscle degradation system, and this may have resulted in maintenance of muscle mass.

Autophagy deficiency has been reported as a cause of sarcopenia. However, because consistent results have not been reported to date with regard to autophagy during aging, we examined how exercise and supplementation affected these parameters. Atg protein extends into the membrane to form autophagosomes, and Beclin1 promotes lysosome binding after autophagosome formation. In this study, Atg3, Atg7, and Atg16L1 levels were significantly lower, whereas Beclin1 expression was significant higher in the Ex+Comb group compared with that in the Con group. These findings suggested that the treatment suppressed the formation of autophagosomes but promoted the formation of autolysosomes, which degrade the produced autophagosomes. In other words, the function of decomposing waste products produced in the muscle was increased. We hypothesized 
that exercise and supplementation could have affected the expression of autophagy-related proteins; however, the results were inconsistent with previous studies. For example, Feng et al. reported that autophagy-related proteins, such as Atg7, Beclin-1, and LC3, increased after 8 weeks of habitual exercise in rats [30] Additionally, Kim et al. reported that in 12-month-old mice, habitual exercise increased LC3-II, Beclin-1, and Atg7 protein expression in plantaris and gastrocnemius muscles, resisting typical age-related decreases [31]. These previous studies examined the effects of exercise alone, and our current findings may have differed because of differences in exercise intensity and animal species. Moreover, few reports have described the effects of HMB on autophagy, and to the best of our knowledge, no reports have evaluated the roles of BG. Therefore, the mechanisms through which these two supplements affect autophagy are still unknown, and further research is needed.

In an aging society, it is necessary to examine how to extend healthy life expectancy, and the health of muscles is also essential. Therefore, the findings of this study are novel findings and suggested that continuous exercise, even that performed at low to moderate intensity, combined with HMB and BG supplementation, could contribute to maintaining muscle health. A summary of our primary findings is shown in Figure S1. Notably, the dose of the supplement used in this study was lower than that in previous studies and was converted to a value close to the recommended intake for humans, with the goal of facilitating translation to human studies. Accordingly, additional studies are needed in human studies to confirm and validate our findings.

\section{Conclusions}

In conclusion, we demonstrated that long-term habitual exercise and combination of HMB plus BG maintained muscle mass and muscle strength against aging in SAMP8 mice. Thus, these changes may be associated with improving mitochondrial biogenesis, enhancing muscle protein synthesis, and stabilizing muscle protein degradation.

Author Contributions: K.A., T.A., T.N., M.T., and H.O. conceived and designed the experiments; K.A., M.K., K.T., and K.H. performed the experiments; K.A. drafted the manuscript; K.A., M.K., K.T., and K.H. analyzed the data; T.S provided the experimental technique; T.A., T.N., M.T., T.S., and K.T. provided critical comments and contributed to the discussion of the results; K.A., M.K., and H.O. edited and revised the manuscript. All authors approved the final version of the manuscript.

Funding: This research was funded by Zenyaku Kogyo Co., Ltd.

Acknowledgments: We thank Jun Shiromoto for helping with the dissection and treadmill training. Additionally, we thank to Yasuko Yoshida for commenting on this study.

Conflicts of Interest: The authors declare no conflicts of interest.

\section{Appendix}

The Graphical Summary is shown in Figure S1.

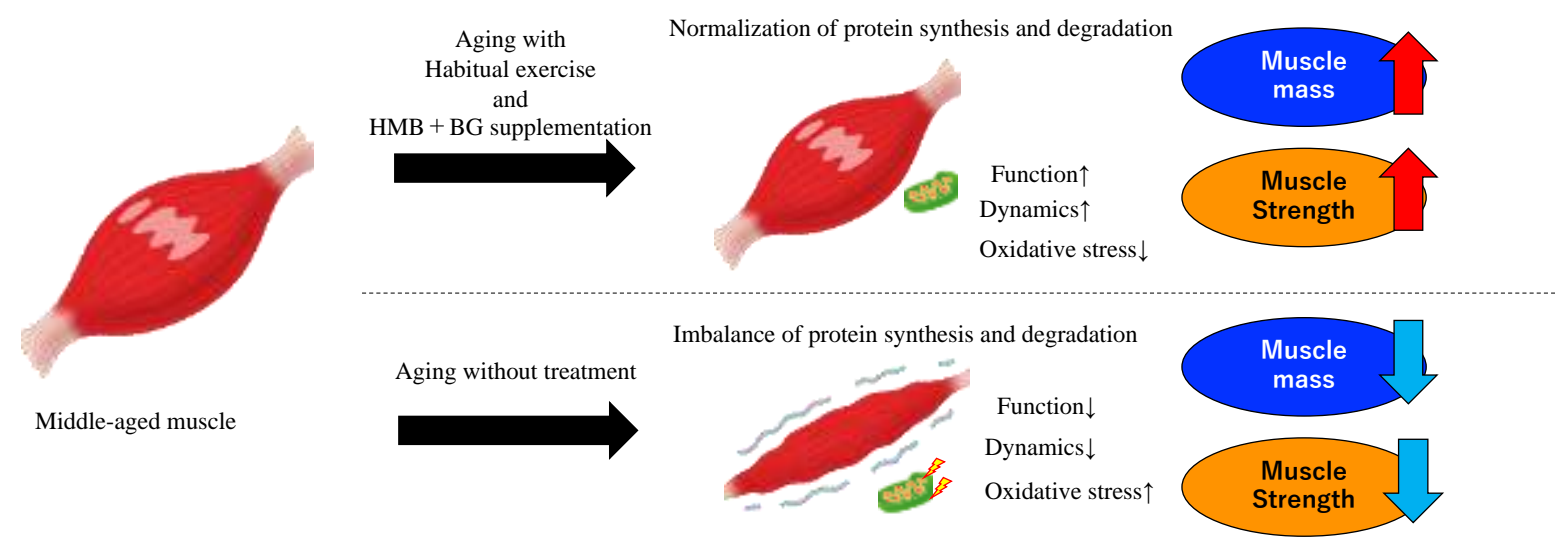


Figure S1. Graphical Summary

\section{References}

1. Rosenberg, I. Summary comments: epidemiological and methodological problems in determining nutritional status of older persons. Am. J. Clin. Nutr. 1989, 50, 1231-1233

2. Bellanti, F.; Romano, A.D.; Lo Buglio, A.; Castriotta, V.; Guglielmi, G.; Greco, A.; Serviddio, G.; Vendemiale, G. Oxidative stress is increased in sarcopenia and associated with cardiovascular disease risk in sarcopenic obesity. Maturitas. 2018, G 109, 6-12.

3. Giorgi, C.; Marchi, S.; Simoes, I.C.M.; Ren, Z.; Morciano, G.; Perrone, M.; Patalas-Krawczyk, P.; Borchard, S.; Jędrak, P.; et al. Mitochondria and reactive oxygen species in aging and age-related diseases. Int. Rev. Cell Mol. Biol. 2018, 340, 209-344.

4. Stefanatos, R.; Sanz, A. The role of mitochondrial ROS in the aging brain. FEBS Lett. 2018, 592, 743-758.

5. Lee, H.; Kim, K.; Kim, B.; Shin, J.; Rajan, S.; Wu, J.; Chen, X.; Brown, M.D.; Lee, S.; Park, J.Y. A cellular mechanism of muscle memory facilitates mitochondrial remodelling following resistance training. J. Physiol. 2018, 596, 4413-4426.

6. Masiero, E.; Agatea, L.; Mammucari, C.; Blaauw, B.; Loro, E.; Komatsu, M. Autophagy is required to maintain muscle mass. Cell Metab. 2009, 10, 507-515.

7. Sopariwala, D.H.; Yadav, V.; Badin, P.M.; Likhite, N.; Sheth, M.; Lorca, S. Long-term PGC1beta overexpression leads to apoptosis, autophagy and muscle wasting. Sci. Rep. 2017, 7, 10237.

8. Kimura, K.; Cheng, X.W.; Inoue, A.; Hu, L.; Koike, T.; Kuzuya, M. $\beta$-Hydroxy- $\beta$-methylbutyrate facilitates PI3K/Akt-dependent mammalian target of rapamycin and FoxO1/3a phosphorylations and alleviates tumor necrosis factor $\alpha /$ interferon $\gamma$-induced MuRF-1 expression in C2C12 cells. Nutr. Res. 2014, 34, 368374.

9. Cuervo, A.M.; Bergamini, E.; Brunk, U.T.; Dröge, W.; French, M.; Terman, A. Autophagy and aging: the importance of maintaining "clean" cells. Autophagy. 2005, 1, 131-140.

10. Capelli, C.; Rittveger, J.; Bruseghini, P.; Calabria, E.; Tam, E. Maximal aerobic power and anaerobic capacity in cycling across the age spectrum in male master athletes. Eur. J. Appl. Physiol. 2016, 116, 1395410 .

11. Tromm, C.B.; Pozzi, B.G.; Paganini, C.S.; Marques, S.O.; Pedroso, G.S.; Souza, P.S. The role of continuous versus fractionated physical training on muscle oxidative stress parameters and calcium-handling proteins in aged rats. Aging Clin. Exp. Res. 2016, 28, 833-841.

12. Ziaaldini, M.M.; Koltai, E.; Csende, Z.; Goto, S.; Boldogh, I.; Taylor, A.W.; Radak, Z. Exercise training increases anabolic and attenuates catabolic and apoptotic processes in aged skeletal muscle of male rats. Exp. Gerontol. 2015, 67, 9-14.

13. Ghosh, S.; Lertwattanarak, R.; Lefort, N.; Molina-Carrion, M.; Joya-Galeana, J.; Bowen, B.; Musi, N. Reduction in reactive oxygen species production by mitochondria from elderly subjects with normal and impaired glucose tolerance. Diabetes. 2011, 60, 2051-2060.

14. Short, K.R.; Bigelow, M.L.; Kahl, J.; Singh, R.; Coenen-Schimke, J.; Raghavakaimal, S.; Nair, K.S. Decline in skeletal muscle mitochondrial function with aging in humans. Proc. Natl. Acad. Sci. U. S. A. 2005, 102, 56185623.

15. Fujita, S.; Rasmussen, B.B.; Cadenas, J.G.; Drummond, M.J.; Glynn, E.L.; Sattler, F.R.; Volpi, E. Aerobic exercise overcomes the age-related insulin resistance of muscle protein metabolism by improving endothelial function and Akt/mammalian target of rapamycin signaling. Diabetes. 2007, 56, 1615-1622.

16. Girón, M.D.; Vílchez, J.D.; Shreeram, S.; Salto, R.; Manzano, M.; Cabrera, E.; Campos, N.; Edens, N.K.; Rueda, R.; López-Pedrosa, J.M.; et al. $\beta$-Hydroxy- $\beta$-methylbutyrate (HMB) normalizes dexamethasone-induced autophagy-lysosomal pathway in skeletal muscle. PLoS One. 2015, 10, e0117520.

17. Stancliffe, A. Role of Beta-hydroxy-Beta-methylbutyrate (HMB) in leucine stimulation of muscle mitochondrial biogenesis. University of Tennessee, Knoxville Trace: Tennessee Research and Creative Exchange. 2012.

18. Kusirisin, W.; Srichairatanakool, S.; Lerttrakarnnon, P.; Lailerd, N.; Suttajit, M.; Jaikang, C. Antioxidative activity, polyphenolic content and anti-glycation effect of some Thai medicinal plants traditionally used in diabetic patients. Med. Chem. 2009, 5, 139-147

19. Rujjanawate, C.; Kanjanapothi, D.; Amornlerdpison, D.; Ponjanagaroon, S. Anti-gastric ulcer effect of Kaempferia paraviflora. J. Ethonopharmacol. 2005, 102, 120-122. 
20. Toda, K.; Hitoe, S.; Takeda, S.; Shimoda, H. Black ginger extract increases physical fitness performance and muscular endurance by improving inflammation and energy metabolism. Heliyon. 2016, 2, e00115.

21. Toda, K.; Takeda, S.; Hitoe, S.; Nakamura, S.; Matsuda, H.; Shimoda, H. Enhancement of energy production by black ginger extract containing polymethoxy flavonoids in myocytes through improving glucose, lactic acid and lipid metabolism. J. Nat. Med. 2016b, 70, 163-172.

22. Wattanathorn, J.; Muchimapura, S.; Tong-Un, T.; Saenghong, N.; Thukhum-Mee, W.; Sripanidkulchai, B. Positive modulation effect of 8-week consumption of Kaempferia parviflora on health-related physical fitness and oxidative status in healthy elderly volunteers. Evid. Based. Complement. Alternat. Med. 2012, 2012, 732816.

23. Takeda, T.; Matsushita, M.; Kurozumi, M.; Takemura, K.; Higuchi, K.; Hosokawa, M. Pathobiology of the senescence-accelerated mouse (SAM). Exp. Gerontol. 1997, 32, 117-127.

24. Wim, D.; Bert, E.; Monique, R.; Peter, H. Soleus muscles of SAMP8 mice provide an accelerated model of skeletal muscle senescence. Exp. Gerontol. 2005, 40, 562-572.

25. Guo, A.Y.; Leung, K.S.; Siu, P.M.F.; Qin, J.H.; Chow, S.K.H.; Qin, L.; Li, C.Y.; Cheung, W.H. Muscle mass, structural and functional investigations of senescence-accelerated mouse. Exp. Anim. 2015, 64, 425-433.

26. Nissen, S.; Sharp, R.; Ray, M.; Rathmacher, J.A.; Rice, D.; Fuller, Jr., J.C.; Connelly, A.S.; Abumrad, N. Effect of leucine metabolite beta-hydroxy-beta-methylbutyrate on muscle metabolism during resistance-exercise training. J. Appl. Physiol. 1996, 81, 2095-2104.

27. Durkalec-Michalski, K.; Jeszka, J. The effect of $\beta$-hydroxy- $\beta$-methylbutyrate on aerobic capacity and body composition in trained athletes. J. Strength Cond. Res. 2016, 30, 2617-2626.

28. Yoshino, S.; Kim, M.; Awa, R.; Kuwahara, H.; Kano, Y.; Kawada, T. Kaempferia parviflora extract increases energy consumption through activation of BAT in mice. Food Sci. Nutr. 2014, 2, 634-637.

29. Sandri, M.; Sandri, C.; Gilbert, A.; Skurk, C.; Calabria, E.; Picard, A.; Walsh, K.; Schiaffino, S.; Lecker, S.H.; Goldberg, A.L. Foxo transcription factors induce the atrophy-related ubiquitin ligase atrogin-1 and cause skeletal muscle atrophy. Cell. 2004, 117, 399-412

30. Feng, Z.; Bai, L.; Yan, J.; Li, Y.; Shen, W.; Wang, Y.; Liu, J. Mitochondrial dynamic remodeling in strenuous exercise-induced muscle and mitochondrial dysfunction: regulatory effects of hydroxytyrosol. Free Radic. Biol. Med. 2011, 50, 1437-1446.

31. Kim, Y.A.; Kim, Y.S.; Oh, S.L.; Kim, H.J.; Song, W. Autophagic response to exercise training in skeletal muscle with age. J. Physiol. Biochem. 2013, 69, 697-705. 